\title{
UPLC-MS/MS analysis and biological activity of the potato cyst nematode hatching stimulant, solanoeclepin $A$, in the root exudate of Solanum spp.
}

\author{
Alessandra Guerrieri ${ }^{1} \cdot$ Kristýna Floková $^{1}$ - Lieke E. Vlaar ${ }^{1}$. Mario L. Schilder ${ }^{1} \cdot$ Gertjan Kramer $^{2}$. \\ Aleksandra Chojnacka ${ }^{3} \cdot$ Yannick R. van Dijk ${ }^{1}$ Harro J. Bouwmeester ${ }^{1} \cdot$ Lemeng Dong $^{1}$ (i)
}

Received: 23 June 2021 / Accepted: 4 October 2021 / Published online: 2 November 2021

(c) The Author(s) 2021

\begin{abstract}
Main conclusion Solanoeclepin A is a hatching stimulant for potato cyst nematode in very low (pM) concentrations. We report a highly sensitive method for the analysis of SolA in plant root exudates using UHPLC-MS/MS and show that there is considerable natural variation in SolA production in Solanum spp. corresponding with their hatching inducing activity.
\end{abstract}

\begin{abstract}
Potato cyst nematode (PCN) is a plant root sedentary endoparasite, specialized in the infection of solanaceous species such as potato (Solanum tuberosum) and tomato (Solanum lycopersicum). Earlier reports (Mulder et al. in Hatching agent for the potato cyst nematode, Patent application No. PCT/NL92/00126, 1996; Schenk et al. in Croat Chem Acta 72:593-606, 1999) showed that solanoeclepin A (SolA), a triterpenoid metabolite that was isolated from the root exudate of potato, induces the hatching of PCN. Its low concentration in potato root exudate has hindered progress in fully understanding its hatching inducing activity and exploitation in the control of PCN. To further investigate the role of SolA in hatching of PCN, the establishment of a highly sensitive analytical method is a prerequisite. Here we present the efficient single-step extraction and UHPLC-MS/MS based analysis for rapid determination of SolA in sub-nanomolar concentrations in tomato root exudate. This method was used to analyze SolA production in different tomato cultivars and related solanaceous species, including the trap crop Solanum sisymbriifolium. Hatching assays with PCN, Globodera pallida, with root exudates of tomato genotypes revealed a significant positive correlation between SolA concentration and hatching activity. Our results demonstrate that there is natural variation in SolA production within solanaceous species and that this has an effect on PCN hatching. The analytical method we have developed can potentially be used to support breeding for crop genotypes that induce less hatching and may therefore display reduced infection by PCN.
\end{abstract}

Keywords Analytical chemistry $\cdot$ Eclepins $\cdot$ Hatching assay $\cdot$ Solid phase extraction (SPE) $\cdot$ Tomato root exudates

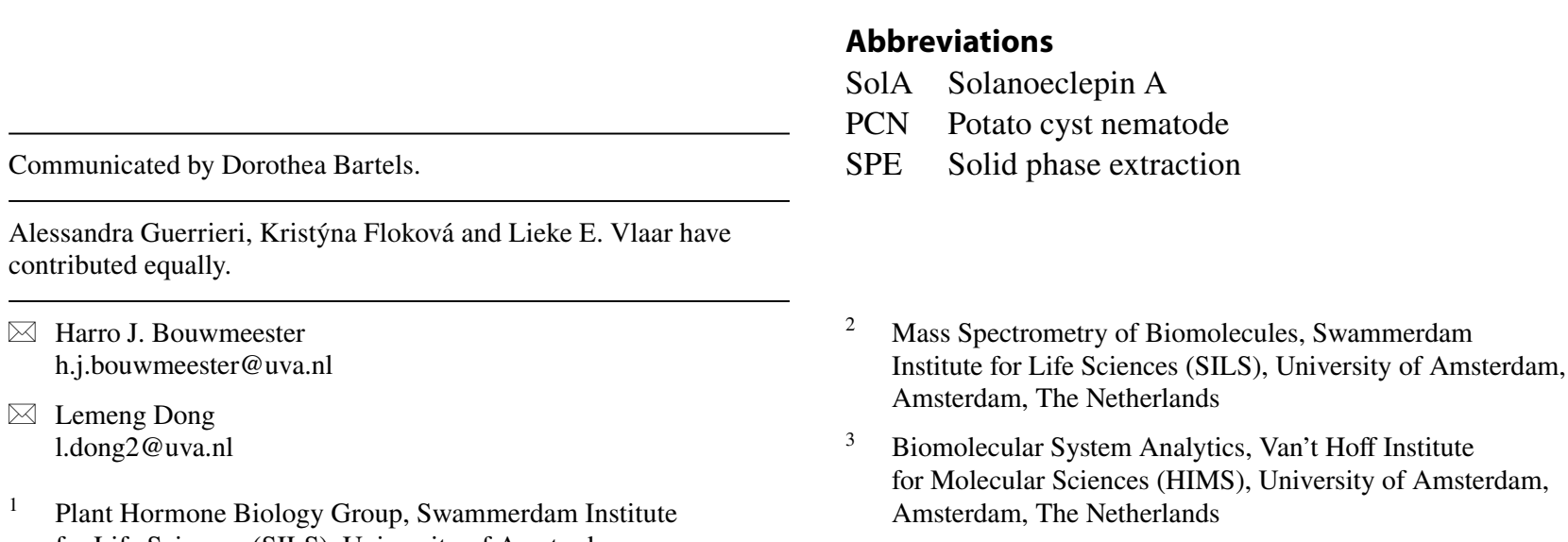

for Life Sciences (SILS), University of Amsterdam,

Amsterdam, The Netherlands 


\section{Introduction}

Potato cyst nematodes (PCNs), Globodera pallida and Globodera rostochiensis, are plant parasitic nematodes and form one of the most damaging pests for solanaceous species, including economically valuable crops such as potato, tomato and eggplant (Perry et al. 2018). These nematodes are called 'cyst nematode' because they form a cyst, the hardened dead body of the female, produced at the end of the life cycle that remains in the soil after the crop is harvested (Perry et al. 2018). The cyst contains hundreds of eggs that are protected against biotic and abiotic stresses for up to 20 years. When a suitable host is nearby, the eggs hatch in response to hatching stimulants produced by the host roots, after which the hatched juveniles penetrate the root and induce a feeding site called syncytium (Bohlmann 2015; Perry et al. 2018).

Since 1922 a great number of studies have been carried out on identifying hatching factors in potato (Calam et al. 1949a; Massey and Neal 1953; Janzen and Van der Tuin 1956; Devine et al. 1996). The most active hatching stimulant so far isolated from potato root exudate was coined solanoeclepin A by Mulder et al. (1996) (Fig. 1). The structure of this compound was further described in 1999 by Schenk et al. (1999). SolA is a triterpenoid acid with a similar structure as the hatching stimulants of soybean cyst nematode, reported in kidney bean, glycinoeclepin
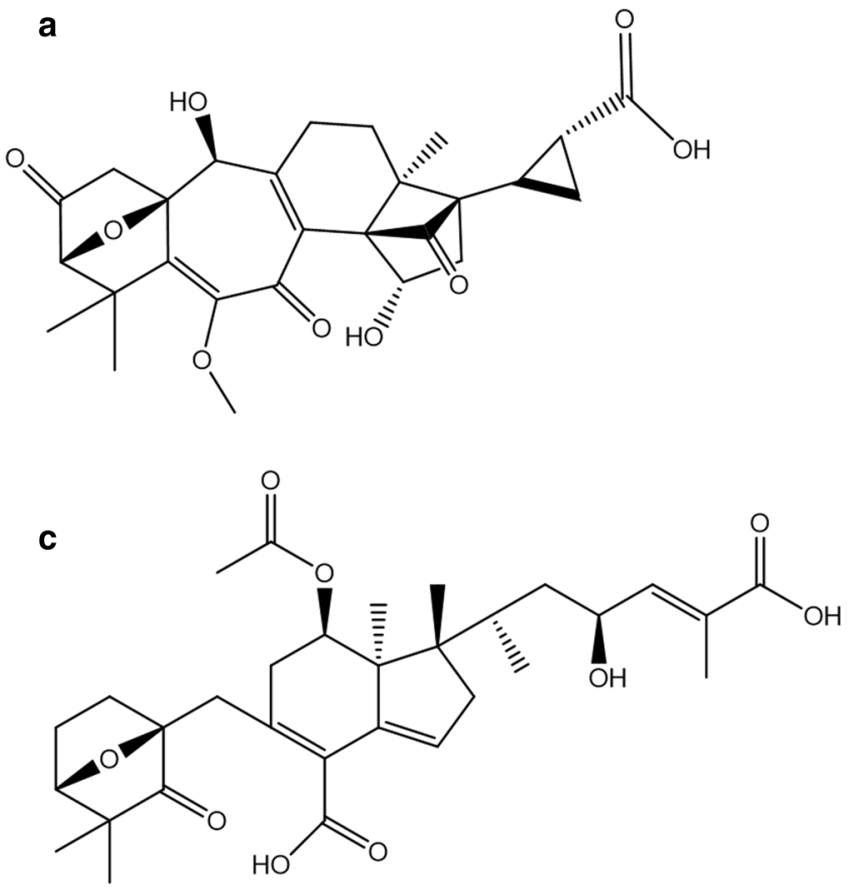

A, B and C (Fig. 1) (Masamune et al. 1982; Fukuzawa et al. 1985).

According to Mulder et al. (1996) SolA was first isolated from the root exudate of 700 potato plants grown in hydroponics. The nutrient solution on which the plants were grown was passed through a column containing Amberlite resin to adsorb the hatching agents, which were subsequently eluted from the column and subjected to a series of purification and fractionation steps. The fractions obtained were tested with hatching assays to determine which fractions induced hatching. The fractions that showed hatching activity were finally combined and concentrated to obtain $245 \mu \mathrm{g}$ of pure hatching agent whose structure was then determined (Mulder et al. 1996). Tanino et al. (2011) reported for the first time the complete chemical synthesis of SolA. The activity of the synthetic SolA was tested in a series of hatching assays with $G$. rostochiensis, and its hatching activity at concentrations as low as $1 \times 10^{-8}-1 \times 10^{-10} \mathrm{~g} \mathrm{ml}^{-1}$ water was confirmed. Moreover the hatching activity was compared to the activity of a tomato root exudate concentrated from 5001 nutrient solution used to grow 12,000 tomato plants in hydroponics (Tanino et al. 2011). The activity of the synthetic SolA was only $65 \%$ of the activity of the tomato root exudate, suggesting the presence of other hatching factors (Tanino et al. 2011). Shimizu et al. (2020) tested the hatching activity of the major steroidal glycoalkaloids in potato and tomato root exudates, on G. rostochiensis. Despite the significant hatching stimulant activity of all the tested compounds, including $\alpha$-solanine, $\alpha$-chaconine and $\alpha$-tomatine,
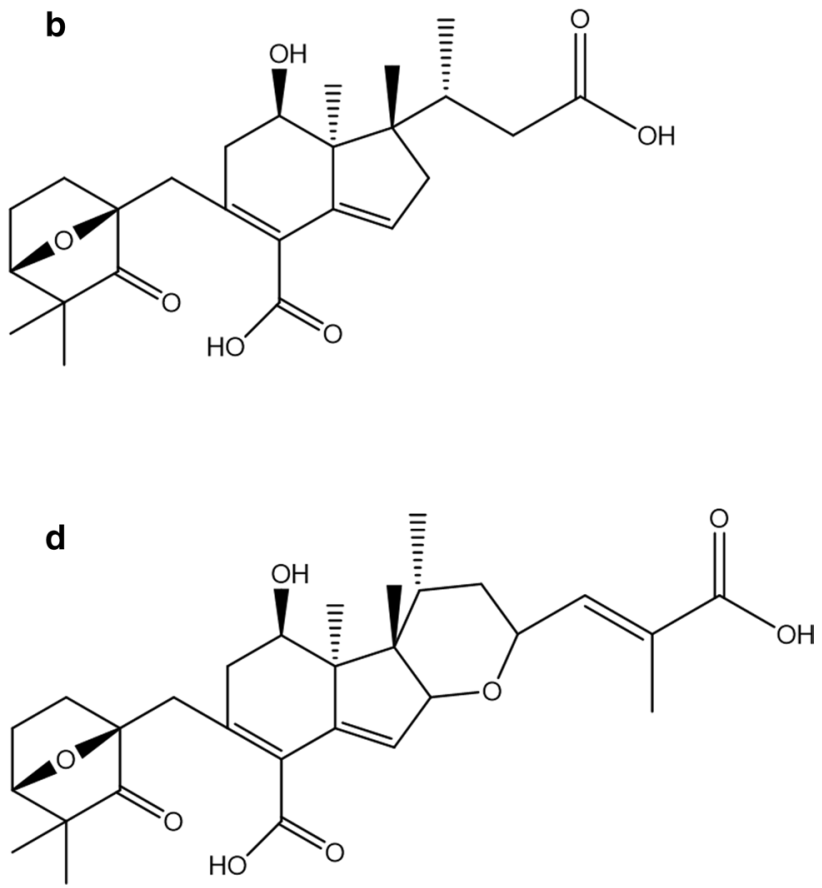

Fig. 1 Structures of known hatching factors for cyst nematodes. a Solanoeclepin A. b Glycinoeclepin A. c Glycinoeclepin B. d Glycinoeclepin C 
however, when compared to SolA the activities were quite weak.

The direct application of 'hatching factors' into the soil to control the spreading of PCN has been proposed, the socalled 'suicide hatch' strategy. For example, Devine and Jones (2000) demonstrated that the exogenous application of hatching factors in the field before planting, resulted in a $50 \%$ reduction in the population of G. rostochiensis, due to the high mortality of the juveniles in the absence of their plant host. The 'hatching factors' were obtained by concentrating tomato and potato root exudates from 7000 and 40 plants grown in hydroponic culture and pots, respectively, using Amberlite resin. A similar approach is the trap-crop strategy, employing a crop that induces hatching of the cyst nematodes but is not a suitable host or is destroyed before the host has completed its life cycle, thus reducing the infestation level (Scholte 2000a). Solanum nigrum and Solanum sisymbriifolium have been proposed as potential trap crops to control PCN (Scholte 2000b). In both the suicide hatch and trap crop approach, it is unknown whether SolA is responsible for the hatching activity, or whether other factors induce the hatching response in potato cyst nematodes. Both Mulder et al. (1996) and Schenk et al. (1999) did not provide insight into the concentration of SolA in potato or tomato root exudates. Thus, it is difficult to provide advice on the precise amount of root exudates to apply to reach the desired effect of PCN control. Likewise, without knowledge of the hatching factor identity and concentration in the root exudate of trap crops it is difficult to optimize this PCN control strategy.

Up to now, one of the indirect methods to quantify SolA is the hatching assay performed on cysts or eggs of PCN (Been and Schomaker 2001). However, this method is not very accurate, since large variation can be introduced by using different nematode species/pathotypes and root exudates (Devine and Jones 2001a; b). As mentioned before, hatching assays can be influenced by the presence of different hatching factors in the root exudates as recently confirmed (Ochola et al. 2020). It has already been reported by Byrne et al. (1998), that hatching factors such as $\alpha$-solanine and $\alpha$-chaconine induce a dosage-dependent response in $G$. rostochiensis. Moreover, at concentrations above $1 \times 10^{-4} \mathrm{M}$, these compounds inhibited hatching (Byrne et al. 1998). Finally, hatching assays lack a standard procedure and require the use of potato cyst nematodes which are quarantine organisms in many countries.

Strikingly, even though there are several indications, there is no direct evidence for the presence of SolA in tomato root exudate. The presence of SolA has so far only been demonstrated in potato root exudates as described in the patent from Mulder et al. (1996). In the patent the method is claimed to be usable also for the isolation of SolA from tomato root exudates. Schenk et al. (1999) reported that their procedure could be used to isolate a compound from tomato root exudate with the same structure as SolA, but it is unclear from their paper which genotype of tomato was used, how it was grown and how many plants were used. A better insight into the presence of SolA in the root exudate of crop genotypes and its role in PCN hatching can open up the possibility for breeding new genotypes of plants with low hatching activity, i.e. a low concentration of hatching factors, including SolA.

Thus, there is a great need to quantify the SolA production in root exudates and further discover the importance of this hatching factor. The techniques so far described in the literature are laborious, including adsorption on active charcoal, ether extraction of acidified aqueous samples and fractionation, and silica gel column chromatography (Calam et al. 1949b; Marrian et al. 1949) and therefore not suitable for routine analysis. Moreover, there is a lack of information on compound loss and recovery. Also, large amounts of plants and root exudate are needed to isolate sufficient SolA.

Therefore, we set out to establish an UHPLC-MS/MSbased method for the fast and sensitive analysis of the SolA concentration in root exudates. Hereto, we used a single-step purification step, employing a mixed-mode anion exchange sorbent, which efficiently pre-concentrates acidic analytes and improves sample purity. With this method, we can quantify SolA in $5 \mathrm{ml}$ of root exudate of a single plant. Using our new approach, we detected and quantified SolA in root exudates of cultivated tomato (Solanum lycopersicum cv Moneymaker and cv MicroTom), their wild relatives (Solanum pennellii, Solanum pimpinellifolium, Solanum habrochaites) and the commonly used trap-crop S. sisymbriifolium. By comparing the SolA concentration in root exudates of different solanaceous species with the hatching response of the PCN G. pallida we show evidence of the importance of SolA in determining the hatching activity of these species.

\section{Materials and methods}

\section{Chemicals and materials}

An authentic standard of solanoeclepin A was kindly provided by Prof. Keiji Tanino (Hokkaido University, Japan). The methanol, acetonitrile, deionized water and formic acid for UPLC-MS/MS and LC-ESI-QTOF-MS analysis were all hypergrade for LC-MS, purchased from Biosolve BV (Valkenswaard, The Netherlands). The $\mathrm{KOH}$ and ammonia for sample extraction and purification were obtained from Merck (Darmstadt, Germany). Deionized (Milli-Q) water was prepared using a water purification system Milli- $\mathrm{Q}^{\circledR}$ (Merck Millipore, Burlington, MA, USA). 


\section{Plant growing and sample collection}

Seeds of tomato (S. lycopersicum cv Moneymaker and MicroTom) were obtained from our greenhouse staff, seeds of the wild tomato species (S. pimpinellifolium, S. pennellii and S. habrochaites accessions LA1777 and PI127826) were obtained from Enza Zaden (Enkhuizen, The Netherlands); seeds from S. sisymbriifolium cv Pion and Quattro, were obtained from Vandinter Research b.v. (Scheemda, The Netherlands).

All seeds were germinated in potting soil in a greenhouse at $24{ }^{\circ} \mathrm{C}, 60 \%$ relative humidity with a $16 \mathrm{~h}$ photoperiod and watered with rainwater. After germination and cotyledon emergence, seedlings were transferred to pots $(15 \mathrm{~cm}$ diameter and $13 \mathrm{~cm}$ height) and grown under the same conditions as for the germination.

Preliminary experiments with tomato showed that SolA can be detected in the root exudate after 2 weeks. It's amount increases until 3 weeks after which it stabilizes until 5 weeks. Thus, 4 weeks old plants were chosen as a standard time point to measure SolA, also because then all the species were in the same phenological stage.

Root exudate collection from five biological replicates was performed on 4-week-old plants: distilled water was poured onto the soil and the flow through from the pot was collected in a beaker until the amount of $300 \mathrm{ml}$ was reached. This amount was previously evaluated to be sufficient to accurately represent the root exudate composition.

Roots of the plants were also collected by washing them and carefully removing the soil particles. The roots were then dried on paper and the fresh weight was recorded (Suppl. Table S2).

Of the $300 \mathrm{ml}$ collected exudate, $50 \mathrm{ml}$ were stored at $4{ }^{\circ} \mathrm{C}$ for further analysis for all the genotypes. For the method development, the remaining $250 \mathrm{ml}$ root exudate of all biological replicates of $S$. lycopersicum cv Moneymaker were pooled and stored for further use.

\section{The stability of solanoeclepin A}

The stability in tested solutions $20 \%, 50 \%$ and $100 \%$ metha$\mathrm{nol} /$ water (v/v) was monitored over 4 weeks. Solutions containing known amount of SolA $\left(5 \mathrm{pmol} \mathrm{ml}^{-1}\right)$ were kept at $-20^{\circ} \mathrm{C}$. Before each measurement, the $0.5 \mathrm{ml}$ of solution $(2.5 \mathrm{pmol})$ was transferred to a new vial and evaporated to dryness using ScanVac vacuum concentrator ScanSpeed 40 (LaboGene, Allerød, Denmark). Samples were reconstituted in $100 \mu \mathrm{l}$ of $20 \%$ methanol (v/v) and analyzed by UPLCMS/MS. The instrument response was normalized by the signal of freshly prepared standard solutions (in duplicates) before each analysis. Following measurements of these solutions contributed to the set of 1,2,3 or 4-week-old samples. Results were calculated as the percentage of mean peak area measured in the set of 1,2,3 or 4-week-old samples in octuplicates, compared to the average of standard area, freshly prepared each week.

The short-term stability of SolA was tested in $100 \%$ methanol, $0.2 \mathrm{M}$ formic acid in methanol and tomato root exudates (TRE, S. lycopersicum cv Moneymaker, prepared as mentioned above). $0.5 \mathrm{ml}$ of these solutions was spiked with $2.5 \mathrm{pmol}$ of SolA and samples were kept for $2 \mathrm{~h}$ at $4{ }^{\circ} \mathrm{C}$. Samples were evaporated in vacuo, reconstituted and analyzed by UHPLC-MS/MS for compound recovery analysis. Additionally, in case of TRE, dried sample matrix was spiked with 2.5 pmol SolA to correct for the effect of the matrix on the signal of the analyte.

\section{Sample extraction and purification}

For sample processing, the root exudates were filtered over filter paper to remove soil particles and the $\mathrm{pH}$ was measured and corrected to around 6.9-7.2 using a $1 \mathrm{M}$ solution of $\mathrm{KOH}$. Five $\mathrm{ml}$ of root exudate was applied to an SPE Oasis ${ }^{\circledR}$ MAX ( 3 cc/60 mg, Waters, Milford, MA, USA) column purchased from Waters Co., pre-conditioned with one column volume $(3 \mathrm{ml})$ of $100 \%$ methanol and activated with one column volume of $5 \% \mathrm{NH}_{4} \mathrm{OH} / \mathrm{H}_{2} \mathrm{O}(\mathrm{v} / \mathrm{v})$.

The retained sample was washed with one column volume of deionized (Milli-Q) water to remove salts and a following wash with one volume of $100 \%$ methanol was applied to elute low-polar contaminants, retained by reversed phase only. SolA elution from the cartridge was achieved with one column volume $0.2 \mathrm{M}$ formic acid in methanol which was collected in a $4 \mathrm{ml}$ glass vial. Samples were evaporated until dryness in vacuo and reconstituted with $100 \mu \mathrm{l}$ of $20 \%$ methanol in water (v/v) for UHPLC-MS/MS analysis.

\section{UHPLC-MS/MS analysis}

The analysis of SolA was performed using a Waters Acquity ultra-high pressure liquid chromatography (UHPLC) ${ }^{\mathrm{TM}}$ I-Class System (Waters) equipped with a binary solvent manager and sample manager, coupled to a Xevo ${ }^{\circledR} \mathrm{TQ}-\mathrm{XS}$ tandem quadrupole mass spectrometer (MS/MS, Waters MS Technologies, Manchester, UK) with electrospray (ESI) ionization interface. The reconstituted sample was filtered using a micro-spin nylon filter $(0.2 \mu \mathrm{m}$ pore size, Thermo Fisher, Waltham, MA, USA). Subsequently, $5 \mu$ was injected onto the reversed-phase UHPLC column (Acquity UPLC ${ }^{\circledR}$ Ethylene Bridged Hybrid (BEH) C18 column, $2.1 \times 100 \mathrm{~mm}$, $1.7 \mu \mathrm{m}$ particle size, Waters), kept at a constant temperature of $40{ }^{\circ} \mathrm{C}$. For the comparison, another UPLC C18 column (Acquity UPLC ${ }^{\circledR}$ Charged Surface Hybrid (CSH) $2.1 \times 100 \mathrm{~mm}, 1.7 \mu \mathrm{m}$ particle size, Waters) was also used. The analyte was eluted at a flow rate of $0.3 \mathrm{ml} \mathrm{min}{ }^{-1}$ with a 9 min linear gradient of $15 \mathrm{mM}$ formic acid/water (A) and 
$15 \mathrm{mM}$ formic acid/acetonitrile (B) with the following elution profile: $0-1 \mathrm{~min}(5 \% \mathrm{~B}), 6 \min (50 \% \mathrm{~B}), 8 \mathrm{~min}(80 \% \mathrm{~B})$, 9 min $(95 \%$ B). At the end of the gradient, the column was washed with $95 \%$ B for $1 \mathrm{~min}$ and finally equilibrated to initial conditions for $2 \mathrm{~min}$. The eluent was introduced into the ESI ion source of the mass spectrometer, operating at optimized settings: capillary/cone voltage (1200/ $30 \mathrm{~V})$, source temperature $\left(120^{\circ} \mathrm{C}\right)$, desolvation temperature $\left(550{ }^{\circ} \mathrm{C}\right)$ and drying gas flow $\left(1000 \mathrm{l} \mathrm{h}^{-1}\right)$. SolA was analyzed in positive mode as $[\mathrm{M}+\mathrm{H}]^{+}$, using diagnostic and confirming precursor-to-product transitions 499>83, > 399, > 315, > 453 with optimized collision energy $30,25,25$, and $20 \mathrm{eV}$, respectively, and collision gas (argon) flow of $0.15 \mathrm{ml} \mathrm{min}^{-1}$. The instrument control, MS data acquisition, and processing were carried out by the MassLynx ${ }^{\mathrm{TM}}$ software, version 4.2 (Waters).

\section{LC-ESI-QTOF-MS analysis}

SolA $(10 \mu \mathrm{M})$ was analyzed using a QTOF MS equipped with a dual-stage trapped ion mobility separation cell (timsTOF pro Bruker Daltonics Inc, Billerica, MA, USA). Sample injection $(40 \mu \mathrm{l})$ and LC separation were performed on an Ultimate RS UHPLC system (Thermo Scientific, Germeringen, Germany) with an Acquity UPLC CSH C18 $130 \AA, 1.7 \mu \mathrm{m}, 2.1 \mathrm{~mm} \times 100 \mathrm{~mm}$ protected by a VanGuard $2.1 \mathrm{~mm} \times 5 \mathrm{~mm}$ of the same material. A gradient from $1 \%$ acetonitrile to $99 \%$ acetonitrile in 18 min was applied at $0.4 \mathrm{ml} \mathrm{min}{ }^{-1}$ (solvent A $0.1 \%$ formic acid in water, solvent B $0.1 \%$ formic acid in acetonitrile), before returning to initial conditions. Eluting compounds were sprayed in positive ion mode by an Apollo II ion funnel ESI source (Bruker Daltonics Inc). Source settings were: capillary voltage $4500 \mathrm{~V}$; end plate offset $500 \mathrm{~V}$; drying temperature $220^{\circ} \mathrm{C}$; desolvation gas (nitrogen) flow $8.01 \mathrm{~min}^{-1}$; nebulizer gas pressure 2.2 bar. Samples were analyzed in timsoff mode, auto MS/ MS settings were: switching threshold 500 cts; cycle time $0.5 \mathrm{~s}$; active exclusion after 3 spectra; release after $0.2 \mathrm{~min}$. Analytes selected for fragmentation were fragmented by collision with nitrogen gas at a collision energy of $30 \mathrm{eV}$. Precursors and fragments were analyzed by the time of flight analyzer using a range of $100-1350 \mathrm{~m} / \mathrm{z}$. Resulting data were analyzed using DataAnalysis ver. 4.3 (Bruker Daltonics).

\section{Fractionation of root exudates}

To determine whether hatching activity and SolA co-eluted, we fractioned a tomato root exudate on UHPLC. Hereto, $1 \mathrm{ml}$ of root exudate from Moneymaker was freeze dried. Salts were precipitated by dissolving the dried exudate in $1 \mathrm{ml}$ of methanol. The sample was centrifuged at $3000 \mathrm{~g}$ for $3 \mathrm{~min}$, and the supernatant was transferred to a clean vial prior to drying by vacuum evaporation. The sample was then re-dissolved in $150 \mu \mathrm{l} 25 \% \mathrm{ACN}$ and injected on UHPLC. Separation of the samples was achieved using the same gradient and same column as used for the analysis. The eluent was fractionated using a Waters fraction manager. The sample was injected 8 times, and the SolA fraction, spanning $30 \mathrm{~s}$ of the chromatogram, was collected from each run and pooled. The fraction was again freeze dried, dissolved in $0.4 \mathrm{ml} 2 \%$ ethanol and tested for hatching activity.

\section{Globodera pallida hatching assay}

G. pallida $\mathrm{D} 383$ pathotype $\mathrm{Pa} 3$ was reared in a greenhouse on potatoes (Solanum tuberosum) and subsequently stored at $4{ }^{\circ} \mathrm{C}$, which broke the diapause, until use for all hatching assays. About 50 first generation cysts were soaked in tap water for 1 week at $20^{\circ} \mathrm{C}$ in the dark. Then, the cysts were carefully opened and the eggs were collected in tap water. The egg suspension was distributed in aliquots of $100 \mu \mathrm{L}$ into the wells of a glass-coated 96-wells plate with 50-100 eggs per well. Subsequently, $100 \mu$ tenfold diluted unpurified root exudate, SolA standard of different concentrations in $2 \% \mathrm{f}$ in tap water, or tap water was added to each well. All conditions were tested in triplicate and concentrations given in the Results section are calculated according to the end concentration in the $200 \mu \mathrm{l}$ in each well. Photos were taken of individual well at the start of the assay, and again after plates were incubated for 7 and 11 days at $20^{\circ} \mathrm{C}$ in the dark. Eggs and hatched juveniles were counted twice on each photo and hatching percentage was calculated according to:

$\left(J_{t 11}-J_{t 0}\right) / J_{t 0} \times 100$,

where $J_{t 11}$ is the number of hatched $J_{2}$ after 11 days of treatment, $J_{t 0}$ is the number of hatched $J_{2}$ at the start of the assay, and $E_{t 0}$ is the number of eggs at the start of the assay.

\section{Statistical analysis}

Comparison of SolA levels in different solanaceous species was carried using a one-way ANOVA analysis in R. Homogeneity of variation and normal distribution of hatching data were verified with Levene's test and the Shapiro-Wilk test, respectively. Multiple pairwise comparison Tukey test in $R$ was used to determine differences in hatching percentages between root exudates of different solanaceous species.

\section{Results}

\section{UHPLC-MS/MS method development}

To analyse SolA levels in complex real samples with sufficient sensitivity, a selective extraction procedure was 
developed and combined with optimized UHPLC-MS/MS analysis. For chromatography two different reversed-phasebased UPLC C18 columns (CSH and BEH) were compared. These polymer-based UPLC columns were successfully used in the analysis of multiple plant hormones (Floková et al. 2020). The additional charge on the surface of the CSH particles would theoretically provide a better peak shape for acidic compounds (Urbanová et al. 2013; Floková et al. 2014). However, in a linear 9 min gradient with $15 \mathrm{mM}$ formic acid in the mobile phase, SolA was detected as a late-eluting broadened asymmetrical peak using the CSH column (Suppl. Fig. S1). Under the same conditions, a 1.5 min shorter retention time and excellent peak shape of the analyte were achieved using the BEH column, which was therefore employed in all further experiments. Using further optimized conditions (see "Materials and methods"), a stable and reproducible retention time was achieved with coefficient of variation $0.43 \%(n=191)$.

Injection of SolA into the UHPLC-MS/MS provides both deprotonated $[\mathrm{M}-\mathrm{H}]^{-}$and protonated $[\mathrm{M}+\mathrm{H}]^{+}$molecular ions of $m / z 497$ and 499, respectively, with a stronger signal in the positive ESI mode (Suppl. Fig. S2). The fragmentation pattern of the positively charged molecular ion was further investigated and appropriate precursor-to-product ion transitions for multiple-reaction monitoring (MRM) analysis were selected. The diagnostic product ion $(\mathrm{m} / \mathrm{z} 399$; molecular formula $\mathrm{C}_{22} \mathrm{H}_{23} \mathrm{O}_{7}$ ) is characterized by the loss of the SolA cyclopropane carboxylic acid side chain (G-ring) and a methyl group. The fragment $(\mathrm{m} / \mathrm{z} 83)$ corresponds to the dehydrated cation of the side chain (G-ring) including a methyl, derived from the E/F ring $\left(\mathrm{C}_{5} \mathrm{H}_{7} \mathrm{O}\right)$ (Suppl. Fig. $\mathrm{S} 3 \mathrm{~b})$. Other detected product ions in the daughter spectra represent the rearranged $\mathrm{ABCD}$ ring system $(\mathrm{m} / \mathrm{z} 315$; molecular formula $\mathrm{C}_{19} \mathrm{H}_{23} \mathrm{O}_{4}$ ) (Suppl. Fig. S3b), the loss of the carboxy group $\left(\mathrm{m} / z, 453\right.$; molecular formula $\mathrm{C}_{26} \mathrm{H}_{29} \mathrm{O}_{7}$ ) and the loss of water $\left(\mathrm{m} / \mathrm{z}, 481\right.$; molecular formula $\left.\mathrm{C}_{27} \mathrm{H}_{29} \mathrm{O}_{8}\right)$
(Fig. 2). The collision energy for each of the selected MRM transitions $499>399,>83,>315,>453$ was optimized and all MS/MS conditions, including settings of the mass spectrometer, such as capillary voltage, source/desolvation temperature, desolvation/collision gas flow and cone voltage as listed in Materials and methods. The accurate mass of these fragments was obtained by liquid chromatography followed by high resolution-mass spectrometry (Suppl. Fig. S4).

\section{Solanoeclepin A extraction and purification procedure}

Sample pre-concentration and clean-up based on solid-phase extraction (SPE) is the strategy to simplify complex sample matrices before compound analysis by UHPLC-MS/ MS. SolA with its rearranged tetranortriterpene structure (Fig. 1a) is a polar molecule in which the methyl enol ether and cyclobutanone moieties could be susceptible to hydrolysis in basic and strong acidic conditions (Tanino et al. 2011).

To design a suitable extraction and purification procedure, we tested the chemical stability of SolA. Considering the polarity of SolA and the sample origin (root exudates), the water-miscible methanol was initially selected as the solubilizing solvent for further sample processing. The stability of SolA was monitored in $100 \%$ methanol and its $50 \%$ and $20 \%$ aqueous solutions (v/v) over 4 weeks. The percentage of methanol was chosen to achieve efficient solubility of the compound for LC-MS, longterm sample stability and to meet compatibility with conditions of reversed-phase based SPE sorbents. Samples were dried in vacuo and compound recovery was analyzed by UHPLC-MS/MS. Due to the absence of an appropriate internal standard, inter-week differences of the instrument response were normalized by weekly analysis of freshly prepared standard solutions (2.5 pmol/sample) in duplicates. The average compound recovery was $87 \%$ with no
Fig. 2 Product-ion spectra of SolA and predicted fragmentation pattern

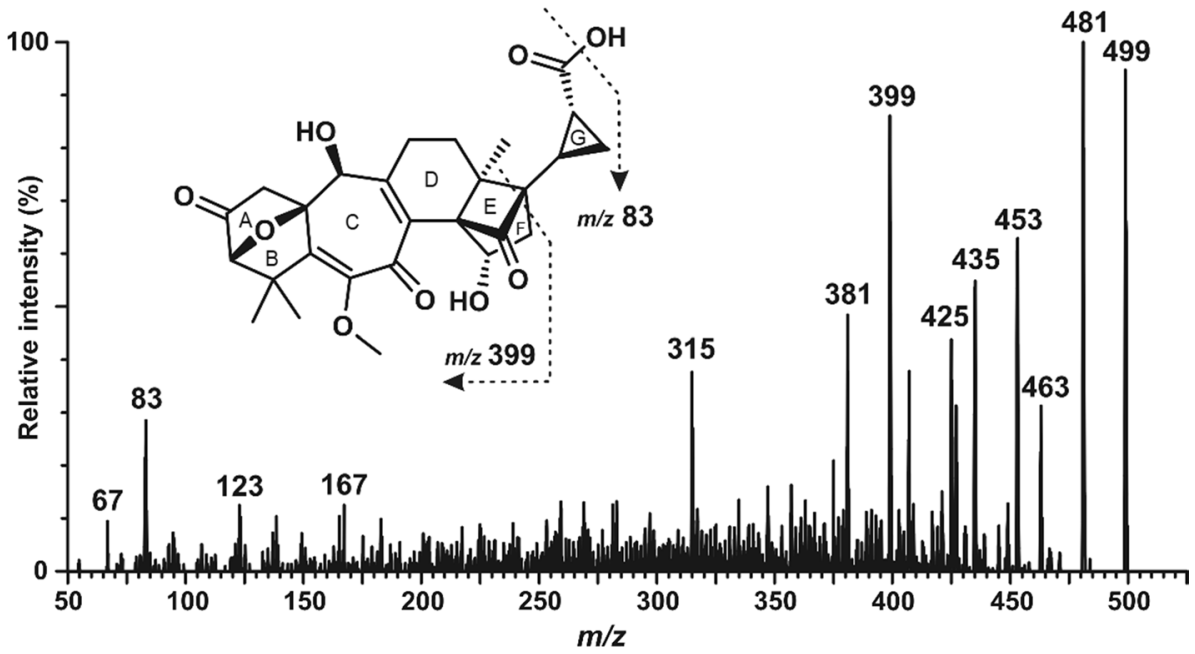


significant differences between individual sets of measurements as well as solutions (Suppl. Fig. S5).

Subsequently, the short-term stability of SolA in root exudates and acidified methanol was analyzed to evaluate critical sample collection and SPE elution conditions. Triplicates of tomato root exudates (TRE) and $0.2 \mathrm{M}$ formic acid in methanol were spiked with 2.5 pmol of SolA and kept at $4{ }^{\circ} \mathrm{C}$ for $2 \mathrm{~h}$. Samples were dried in vacuo and analyzed for compound recovery. The response of SolA, spiked in the dry sample matrix, was analyzed to allow for compensation of the matrix effect on ionization efficiency. Spiked SolA loss in the background of tomato root exudate was $14.1 \%$. The presence of $0.2 \mathrm{M}$ formic acid in methanol slightly decreased SolA recovery by $8 \%$ compared to pure methanol (Suppl. Table S1).

The presence of the carboxylic group in the structure of SolA provides an opportunity for selective analyte purification through anion exchange chromatography. Therefore, the polymer-based SPE Oasis ${ }^{\circledR}$ MAX (Waters), combining reversed phase with anion exchange retention mechanisms, were used for SolA isolation. Tomato root exudates of which the $\mathrm{pH}$ was adjusted to around 6.9-7.2, were applied on the sorbent that was pre-conditioned with methanol and activated with $5 \%$ aqueous ammonia. The column was then washed with one column volume of water to remove salts. The following wash with $100 \%$ methanol was applied to elute low-polar contaminants, retained by reversed phase only. SolA elution was achieved with 0.2 M formic acid in methanol (Suppl. Fig. S6).

To evaluate the extraction recovery of this sample preparation procedure, quadruplicates of TRE $(5 \mathrm{ml})$ were spiked with $2.5 \mathrm{pmol}$ of SolA and further purified using the Oasis ${ }^{\circledR}$ MAX cartridge (Waters). Samples were evaporated to dryness, reconstituted in $100 \mu \mathrm{l}$ of $20 \%$ methanol/water (v/v) and analyzed by UHPLC-MS/MS. Since an internal standard was not available the effect of the matrix on instrument ionization efficiency was analyzed by comparing the instrument response with the SolA standard, spiked in the dry purified sample matrix. The SolA in the presence of sample matrix was recovered after SPE from 90.3\% (Suppl. Table S1). For comparison, we examined the SolA extraction recovery also from water and obtained only $6.1 \%$ loss. These data suggest a slight decrease in the extraction efficiency in the presence of sample contaminants. Based on these results we concluded that the purification protocol using Oasis ${ }^{\circledR}$ MAX sorbent is suitable for SolA extraction from tomato root exudates. The hatching activity of partially purified (with Oasis ${ }^{\circledR}$ MAX cartridge) root exudate of S. lycopersicum cv. Moneymaker was evaluated and was not different from the crude root exudate (Suppl. Fig. S7).

\section{Method validation}

To determine the sensitivity parameters of the newly developed UHPLC-MS/MS method, a 13-point calibration curve was constructed based on the response areas of the serially diluted authentic standard, injected in quadruplicate. The amount of analyte ranged between $240 \mathrm{amol}$ and $1 \mathrm{pmol}$ (Suppl. Fig. S8a). The instrument detection limit was defined as three times signal-to-noise ratio $(\mathrm{S} / \mathrm{N})$ and this corresponds to $1 \mathrm{fmol}$ of injected SolA. The response was linear over four orders of magnitude with a correlation coefficient of 0.998 and the limit of quantitation 0.002 pmol per injection (Suppl. Fig. S8a, b).

The effectiveness of the method was further evaluated by standard addition using tomato root exudates. Samples ( $5 \mathrm{ml}$, quadruplicate) were spiked with the authentic standard of SolA at two concentration levels (1 and $2.5 \mathrm{pmol}$ ) prior to SPE purification. Purified samples were analyzed by UHPLC-MS/MS and SolA concentrations were calculated individually using two sets of 8-point calibration curves ( $7.8 \mathrm{fmol}-1 \mathrm{pmol}$ ), prepared in blank solvent (external calibration) and purified sample matrix (matrix-matched calibration). Non-spiked extracts of tomato root exudates were analyzed to subtract endogenous SolA levels from the amount of added standard. The overall analytical precision for both spiked concentration levels calculated from external calibration was determined as $4.65 \%$ relative standard deviation (RSD) (Table 1). The method accuracy was assessed as $92.5 \%$ and $79 \%$ of the correct 1 and 2.5 pmol concentration levels, respectively. Both, method precision and accuracy, were improved using matrix-matched calibration that compensates for the ion suppression in the presence of sample matrix (Table 1).

\section{Solanoeclepin A is produced by several different solanaceous species}

Having established an effective SPE protocol for SolA extraction, we quantified the SolA concentrations in root exudates of cultivated tomato (S. lycopersicum cv. Moneymaker), model tomato genotype MicroTom, three wild tomato relatives ( $S$. pimpinellifolium, $S$. pennellii and $S$. habrochaites accessions LA1777 and PI127826) and two cultivars of the trap crop S. sisymbriifolium cv. Pion and Quattro. SolA was detected in all these genotypes (Fig. 3, Suppl. Fig. S8c). There was a significant difference in the SolA production by the two accessions of $S$. habrochaites, with the highest level of SolA produced by PI127826 (67.6 pmol g ${ }^{-1}$ FW) (Fig. 3 and Suppl. Table. S2). S. sisymbriifolium cv. Quattro produced the lowest amount of SolA with a concentration below $2.83 \mathrm{pmol} \mathrm{g}^{-1} \mathrm{FW}$. On the other hand, no significant difference in SolA production of S. sisymbriifolium cv. Pion, S. pennelli and S. lycopersicum 
Table 1 Method validation

\begin{tabular}{llllc}
\hline Tomato root exudates & $\begin{array}{l}\text { Spiked } \\
\text { amount } \\
(\mathrm{pmol})\end{array}$ & $\begin{array}{l}\text { Determined content } \\
(\mathrm{pmol}) \pm \text { Standard devia- } \\
\text { tion }\end{array}$ & $\begin{array}{l}\text { Precision } \\
\text { (\% RSD) }\end{array}$ & Accuracy (\% bias) \\
\hline External calibration & 1 & $0.93 \pm 0.04$ & 4.72 & -7.44 \\
Matrix-matched calibration & 1 & $1.97 \pm 0.09$ & 4.59 & -21.04 \\
& 2.5 & $0.98 \pm 0.01$ & 0.99 & -2.30 \\
Extraction solvent & & $2.11 \pm 0.05$ & 2.21 & -15.47 \\
External calibration & 1 & $1.08 \pm 0.08$ & 7.71 & 7.81 \\
& 2.5 & $2.37 \pm 0.11$ & 4.84 & -5.19 \\
\hline
\end{tabular}

The precision and accuracy of SPE sample preparation was calculated using an 8-point calibration curve, prepared in $20 \%$ methanol/water, v/v (external calibration) and purified samples of tomato root exudates (reconstituted in $20 \%$ methanol/water, v/v; matrix-matched calibration). Tomato root exudates $(5 \mathrm{ml})$ and control samples of extraction solvent $(5 \mathrm{ml}$ of water) were spiked with 1 and 2.5 pmol of SolA standard. Samples were purified using Oasis ${ }^{\circledR}$ MAX SPE sorbent and analyzed by UHPLC-MS/MS. All experiments were performed in quadruplicates. The method's precision is determining relative standard deviation (\% RSD) for each spiked level. The accuracy is assessed as difference of determined content from spiked content in percentage (\% bias)

cv MicroTom. Surprisingly, both cultivars of the trap crop produced less SolA than the commercial tomato cultivar Moneymaker, of which the production is comparable to the wild S. pimpinellifolium $\left(11.6-28.4 \mathrm{pmol} \mathrm{g}^{-1} \mathrm{FW}\right)$. The complete dataset is shown in the Supplemental Table S2.

\section{Hatching of G. pallida eggs under influence of root exudates of solanaceous species}

The effect of the root exudates of these solanaceous species on hatching of G. pallida eggs was evaluated using a hatching bioassay (Suppl. Fig. S6, Fig. 4a). Crude exudates were diluted tenfold, since previous results showed that this provides an optimum concentration for a reproducible hatching assay (Suppl. Fig. S9). There was a relatively large variation between the replicates of root exudate samples from the same species (Suppl. Fig. S6). However, all root exudate replicates showed higher hatching compared to tap water (Suppl. Fig. S6). SolA standard solutions of 0.5 and $5 \mathrm{nM}$ showed comparable hatching ( $\sim 5 \%$ and $75 \%$, respectively) as the most potent root exudates (Suppl. Fig. S6).

There is a significant difference in induced hatching between two groups of exudates: the root exudates of $S$. lycopersicum cv. Moneymaker, S. pimpinellifolium, S. habrochaites acc. LA1777, S. habrochaites acc. PI127826 and S. sisymbriifolium cv. Pion induced higher hatching than those of S. pennellii, S. sisymbriifolium cv. Quattro and $S$. lycopersicum cv. MicroTom (Fig. 4a). The commercial cultivar $S$. lycopersicum cv. Moneymaker induced the highest mean hatching rate, whereas $S$. lycopersicum cv. MicroTom induced the lowest mean hatching rate. Interestingly, the widely used trap crop $S$. sisymbriifolium $\mathrm{cv}$. Pion induced a mean hatching rate of around $45 \%$, which signifies its suitability as a trap crop (Dandurand and Knudsen 2016).
Hatching rates induced by root exudates seem to depend to some extent on their SolA content although this is difficult to conclude as in genotypes $S$. pimpinellifolium, S. habrochaites acc. PI127826 and LA1777 and in S. lycopersicum $\mathrm{cv}$. Moneymaker hatching rate is saturated (Fig. $4 \mathrm{a}-\mathrm{c}$ ). Furthermore, other hatching factors seem to be playing a role, since the non-saturated root exudates (S. sisymbriifolium cv. Pion, S. lycopersicum MicroTom, S. pennelli and S. sisymbriifolium cv. Quattro) that contain only low SolA concentrations, still induce relatively high hatching. For example, the root exudate of $S$. lycopersicum cv. MicroTom induced around $40 \%$ hatching while it contains only about $5 \mathrm{pM}$ SolA, a concentration that, as pure standard, induces no significant hatching compared with water (Fig. 4b). Similar observations hold for root exudates of S. pennelli and S. sisymbriifolium cv. Quattro, which contain around 5 and $1 \mathrm{pM}$ SolA, respectively (Fig. 4a). Nevertheless, correlation analysis of hatching and SolA concentration in the root exudate yielded a positive, logarithmic correlation (Fig. 4c). Likely, the low $R^{2}$ can be explained by the fact that hatching with some exudates was saturated and that there may also be other factors (stimulants and/or inhibitors) other than SolA in the root exudates that affect the hatching rate and therefore the correlation between SolA concentration and hatching. To get a more thorough idea of how much hatching is caused solely by SolA from root exudates, the fraction containing SolA was collected from S. lycopersicum cv. Moneymaker and tested for hatching activity (Suppl. Fig. S10). This fraction was able to hatch almost as much eggs as crude root exudate and a standard solution of SolA of $0.5 \mathrm{nM}$. Hence, overall, there is a reliable positive correlation between SolA concentration and hatching of G. pallida eggs making SolA concentration a good predictor of hatching (Fig. 4c). 

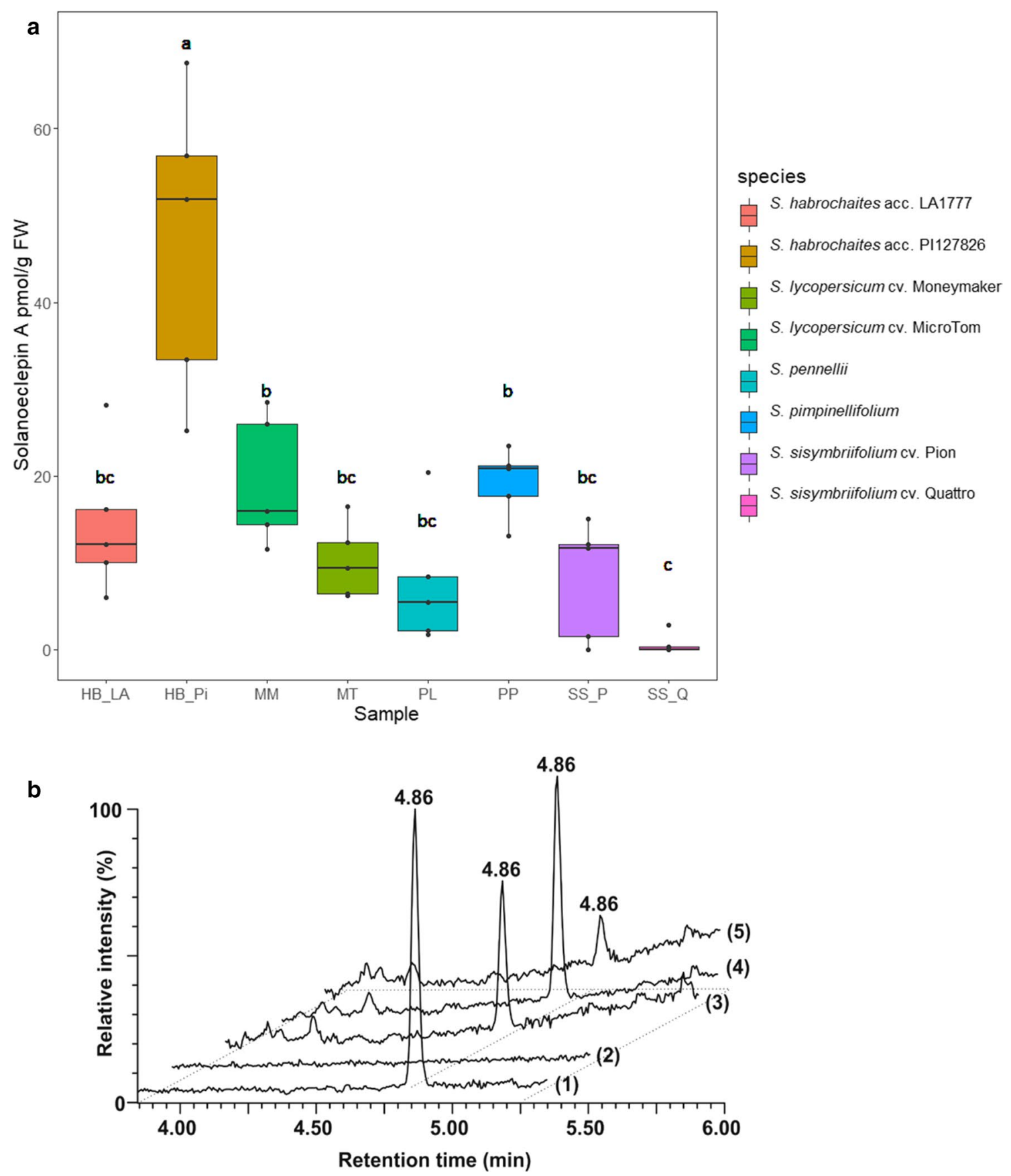

Fig. 3 a SolA concentration in root exudates of different Solanaceae species, S. habrochaites acc. LA177 (HB_LA), S. habrochaites acc. PI127826 (HB_Pi), S. pennellii (PL) and S. pimpinellifolium (PP), tomato cultivars: S. lycopersicum cv. Moneymaker (MM) and MicroTom (MT) and trap crop: Solanum sisymbriifolium cv. Pion (SS_P) and cv. Quattro (SS_Q). The concentration is expressed in $\mathrm{pmol} / \mathrm{gram}$ of fresh root weight $\left(\mathrm{pmol} \mathrm{g}^{-1} \mathrm{FW}\right)$. Black dots represent SolA concentration from each biological replicate. Samples were ana-

\section{Discussion}

The present study established, for the first time, an efficient and simple single-step extraction method of SolA lyzed in five replicates, median (line within the box), first and third quartiles (box), non-outlier range (whiskers), and outliers (dot) are shown. Different letters indicate statistically significant differences between samples using a one-way ANOVA analysis. b LC-MS chromatograms of SolA standard (1), negative control (2) and real samples of root exudates obtained from S. lycopersicum cv. Moneymaker (3), S. habrochaites acc. PI127826 (4) and S. sisymbriifolium cv. Quattro (5)

from root exudates as well as an ultra-sensitive UPLC MS/ MS method to quantify SolA content in plant root exudates. Several attempts of purification and characterization of PCN hatching factors from potato and tomato root 

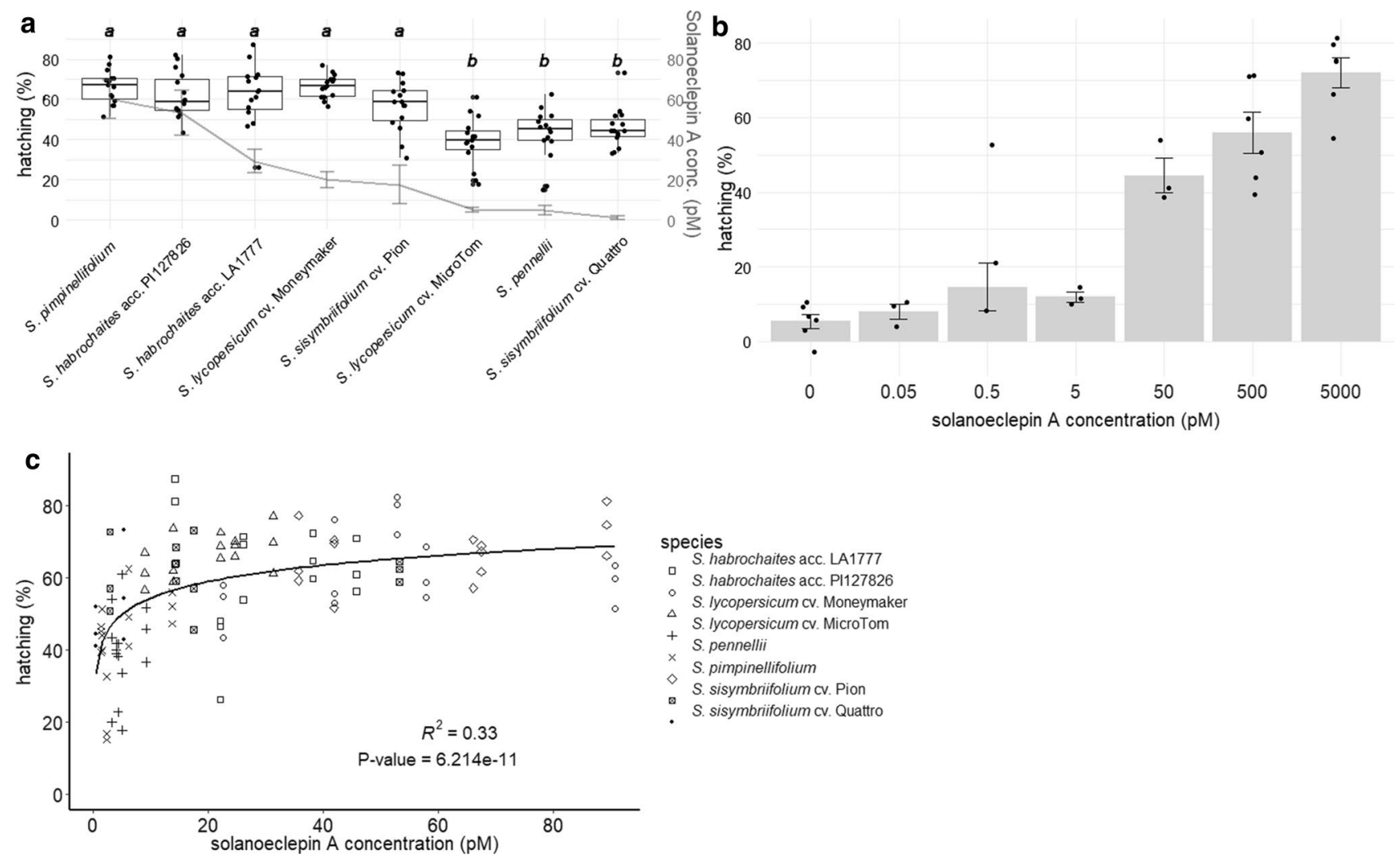

Fig. 4 a Boxplots of hatching of G. pallida eggs treated with root exudates of solanaceous species. Significant differences $(P<0.05)$ are indicated with a and $b$. The line graph shows the SolA concentrations in the root exudates with error bars (standard error, $n=5$ ). b Hatching percentages of $G$. pallida eggs treated with standard solutions of SolA. Bars indicate the mean hatching induced by the respective treatment, error bars indicate standard error, black dots are individual measurements. One outlier at $0.5 \mathrm{pM}$ was ignored in the calculation

exudates have already been reported in the literature from the early 1940s. For example, Calam et al. (1949a,b) proposed a method for the preparation of solid concentrates of PCN hatching factor that included the collection of 1001 of root exudates from 4000 to 5000 plants. The hatching factors in the root exudates were subsequently absorbed on charcoal followed by complicated and time-consuming elution and evaporation steps to obtain a brown powder, which contained $60-70 \%$ of the active factor present in the root exudates (Calam et al. 1949a,b). Mulder et al. (1996) described a complicated procedure to isolate $245 \mu \mathrm{g}$ of SolA from the root exudate of 700 potato plants. Subsequently, Schenk et al. (1999) reported that they managed to purify $200 \mu \mathrm{g}$ of pure SolA from crude potato root exudate, but the experimental procedure by which this result was achieved was not included. Later studies have only reported the method for chemical synthesis of SolA, without investigating the extraction procedure of this compound from plant root exudates (Tanino et al. 2011). of the mean and standard error. c Mean percentages of hatching of $G$. pallida eggs as induced by root exudates of solanaceous species $(S$. pimpinellifolium, S. habrochaites acc. PI127826 and acc. LA1777, S. lycopersicum cv. Money maker and cv. MicroTom, S. sisymbriifolium cv. Pion and cv. Quattro, and S. pennellii) described in this paper, of which the SolA content was known. Line shows the log relationship between SolA concentration and hatching of G. pallida eggs. The $P$-value indicates that the $R^{2}$ value of 0.33 is significant

The method developed here was subsequently used on several different solanaceous species, showing that the method is suitable for investigating the natural variation in SolA levels in tomato, but also other solanaceous species such as wild tomato relatives and the trap crop S. sisymbriifolium. Interestingly, we found significant differences between different accessions of the same species such as $S$. habrochaites acc. PI127826 and acc. LA1777. This result warrants further investigation as the two accessions come from the same center of origin in Peru. Indeed, it has been shown that different $S$. habrochaites accessions can display large chemical diversity, for example in terpenoid production the glandular trichomes (Gonzales-Vigil et al. 2012). Since the method presented here is relatively easy to use, sensitive and fast, it can be applied in high-throughput screening approaches to select genotypes that produce lower amounts of hatching stimulant and can potentially be used to breed for reduced PCN infection. 
Using our new method, we showed that root exudate of the trap crop $S$. sisymbriifolium contains SolA and the hatching assay confirmed its hatching activity for PCN reported before (Scholte 2000b). This finding is different from the results presented by Sasaki-Crawley et al. (2010), who suggested that $S$. sisymbriifolium produces other hatching stimulants, not SolA. This discrepancy is likely caused by the improved analytical method that we have developed and/or by the analysis of different cultivars. Indeed, of the two cultivars that we analyzed, only one is producing a clearly detectable amount of SolA. Our new method opens up the possibility to investigate and optimise the efficiency of the cultivars that are currently used as trap crop to prevent the spreading of PCN.

In the present study, we analyzed root exudates at one time point. For future experiments it could be interesting to also investigate the SolA concentration in root exudates collected at different timepoints and/or different plant phenological stages.

The hatching assay, using eggs of G. pallida, showed that SolA is extremely active in sub-nM concentrations, which is much lower than the values reported for glycoalkaloid hatching stimulants such as $\alpha$-tomatine, which induces only average hatching rates at $\mu \mathrm{M}$ concentrations (Shimizu et al. 2020). Furthermore, from our results, $G$. pallida seems to be equally sensitive to SolA compared to G. rostochiensis and G. tabacum, which were studied by Sakata et al. (2020). However, the same study showed a maximum hatching rate of $20 \%$ by SolA in G. pallida, which is much lower than what we find here. Possibly, Sakata et al. (2020) used a different pathotype of G. pallida, which could explain this discrepancy.

Interestingly, the hatching rates induced by the root exudates from different species and genotypes quite well correlate with their SolA concentration and matches the hatching rates obtained with pure SolA, although a matrix effect seems to be present that increases hatching of SolA in root exudates. Indeed, for some root exudates, such as for S. lycopersicum cv. MicroTom, S. pennelli and S. sisymbriifolium cv. Quattro, the SolA levels are too low to explain their hatching rates of $\sim 40-50 \%$. The root exudates of these genotypes contain 1-5 pM SolA, whereas $40 \%$ hatching is only achieved by $50 \mathrm{pM}$ SolA (Fig. 4b). Hence, the root exudate probably contains additional, unknown, hatching stimulants, causing a high hatching rate despite the low SolA concentration. As already discussed, tomatidine and $\alpha$-tomatine may contribute to induce hatching, but are not very effective and alone cannot explain the hatching rates obtained (Shimizu et al. 2020). Other host species of PCN such as potato, are known to produce a range of hatching factors as well, like $\alpha$-chaconine, $\alpha$-solanine and solanidine (Stobiecki et al. 2003; Shakya and Navarre
2008), which induce hatching in G. rostochiensis, but only at $\mathrm{nM}$ concentrations (Ochola et al. 2020).

Moreover, root exudates may also contain hatching inhibitors that can interfere with the correlation between SolA and hatching rate. Finally, some of the other hatching stimulating compounds described in the literature were shown to induce hatching according to an optimum curve, and at very high concentrations act as inhibitors (Devine et al. 1996; Byrne et al. 1998). Further work should characterize the full spectrum of bioactive compounds in the root exudate that together determine the hatching of PCN.

In conclusion, we developed an efficient method for the analysis of SolA in root exudate and showed that there is substantial natural variation in its production by cultivated and wild tomato genotypes and the trap crop $S$. sisymbriifolium. Hatching assays revealed that there is a significant positive correlation between SolA concentration and hatching activity. The analytical method we have developed can potentially be used to support breeding for crop genotypes that induce less hatching and may therefore display reduced infection by PCN.

Author contribution statement Analytical chemical experiments were designed by KF, MLS, GK and AC and executed by KF, AC, YR van D and AG; growth, harvest and processing of plant samples was designed and executed by AG; hatching assays were designed and executed by LEV; study execution, data collection and preparation of the original draft was carried out by AG, KF, MLS, GK, and LEV. The corresponding authors, LD and HJB, participated in the research design, conceptualization, project administration, funding acquisition, project supervision, review and editing of the manuscript. All authors have read and agreed to the published version of the manuscript.

Supplementary Information The online version contains supplementary material available at https://doi.org/10.1007/s00425-021-03766-2.

Acknowledgements We thank Professor Keiji Tanino (Faculty of Science, Hokkaido University, Sapporo, Japan), for generously providing SolA standard. We thank Enza Zaden (Enkhuizen, The Netherlands) and Vandinter Research b.v. (Scheemda, The Netherlands) for providing the seeds used in this work. We are grateful to Dr. Aska Goverse of the Laboratory of Nematology (Wageningen University and Research) for supplying the cysts used in this study. This research was supported by the ERC (Advanced grant CHEMCOMRHIZO, 670211 to HJB), the EU (Marie Curie grant NemHatch, 793795 to LD) and Dutch Research Council (NWO-TTW grant 16873 Holland Innovative Potato, to H.B. and L.D.).

Data availability The datasets generated during and/or analyzed during the current study are available from the corresponding author on reasonable request. 
Open Access This article is licensed under a Creative Commons Attribution 4.0 International License, which permits use, sharing, adaptation, distribution and reproduction in any medium or format, as long as you give appropriate credit to the original author(s) and the source, provide a link to the Creative Commons licence, and indicate if changes were made. The images or other third party material in this article are included in the article's Creative Commons licence, unless indicated otherwise in a credit line to the material. If material is not included in the article's Creative Commons licence and your intended use is not permitted by statutory regulation or exceeds the permitted use, you will need to obtain permission directly from the copyright holder. To view a copy of this licence, visit http://creativecommons.org/licenses/by/4.0/.

\section{References}

Been TH, Schomaker CH (2001) Ways to improve the accuracy of hatching tests for Globodera spp. with special emphasis on nematicide trials. Nematology 3:773-795. https://doi.org/10.1163/ 156854101753625290

Bohlmann H (2015) Introductory chapter on the basic biology of cyst nematodes. Adv Bot Res 73:33-59. https://doi.org/10.1016/BS. ABR.2014.12.001

Byrne J, Twomey U, Maher N et al (1998) Detection of hatching inhibitors and hatching factor stimulants for golden potato cyst nematode, Globodera rostochiensis, in potato root leachate. Ann Appl Biol 132:463-472. https://doi.org/10.1111/j.1744-7348.1998. tb05222.x

Calam CT, Raistrick H, Todd AR (1949a) The potato eelworm hatching factor. 1 . The preparation of concentrates of the hatching factor and a method of bioassay. Biochem J 45:513-519. https://doi.org/ 10.1042/bj0450513

Calam CT, Todd AR, Waring WS (1949b) The potato eelworm hatching factor. 2. Purification of the factor by alkaloid salt fractionation. Anhydrotetronic acid as an artificial hatching agent. Biochem J 45:520-524. https://doi.org/10.1042/bj0450520

Dandurand LM, Knudsen GR (2016) Effect of the trap crop Solanum sisymbriifolium and two biocontrol fungi on reproduction of the potato cyst nematode, Globodera pallida. Ann Appl Biol 169:180-189. https://doi.org/10.1111/aab.12295

Devine KJ, Jones PW (2000) Response of Globodera rostochiensis to exogenously applied hatching factors in soil. Ann Appl Biol 137:21-29. https://doi.org/10.1002/andp.19003060204

Devine KJ, Jones PW (2001a) Potato cyst nematode hatching activity and hatching factors in inter-specific Solanum hybrids. Nematology 3:141-149. https://doi.org/10.1163/156854101750236268

Devine KJ, Jones PW (2001b) Effects of hatching factors on potato cyst nematode hatch and in-egg mortality in soil and in vitro. Nematology 3:65-74. https://doi.org/10.1163/156854101300106900

Devine KJ, Byrne J, Maher N, Jones PW (1996) Resolution of natural hatching factors for golden potato cyst nematode, Globodera rostochiensis, in potato root leachate. Ann Appl Biol 129:323-334. https://doi.org/10.1111/j.1744-7348.1996.tb05755.x

Floková K, Tarkowská D, Miersch O et al (2014) UHPLC-MS/MS based target profiling of stress-induced phytohormones. Phytochemistry 105:147-157. https://doi.org/10.1016/j.phytochem. 2014.05.015

Floková K, Shimels M, Andreo Jimenez B et al (2020) An improved strategy to analyse strigolactones in complex sample matrices using UHPLC-MS/MS. Plant Methods 16:1-17. https://doi.org/ 10.1186/s13007-020-00669-3

Fukuzawa A, Matsue H, Ikura M, Masamune T (1985) Glycinoeclepins $\mathrm{B}$ and $\mathrm{C}$, nortriterpenes related to glycinoeclepin A. Tetrahedron Lett 26:5539-5542
Gonzales-Vigil E, Hufnagel DE, Kim J et al (2012) Evolution of TPS20-related terpene synthases influences chemical diversity in the glandular trichomes of the wild tomato relative Solanum habrochaites. Plant J 71:921-935. https://doi.org/10.1111/j.1365313X.2012.05040.x

Janzen GJ, Van der Tuin F (1956) The unknown hatching agent for the potato root-eelworm. Nematologica 1:126-137. https://doi.org/ 10.1163/187529256X00500

Marrian DH, Russell PB, Todd AR, Waring WS (1949) The potato eelworm hatching factor. 3. Concentration of the factor by chromatography. Observations on the nature of eclepic acid. Biochem J 45:524-528. https://doi.org/10.1042/bj0450524

Masamune T, Anetai M, Takasugi M, Katsui N (1982) Isolation of a natural hatching stimulus, glycinoeclepin $\mathrm{A}$, for the soybean cyst nematode. Nature 297:495-496. https://doi.org/10.1038/297495a0

Massey LM, Neal LA (1953) Investigations concerning the hatching factor of the golden nematode of potatoes, Heterodera rostochiensis Wollenweber on JSTOR. J Washingt Acad Sci 43:396-401

Mulder JG, Diepenhorst P, Plieger P, Brüggemann-Rotgans IEM (1996) Hatching agent for the potato cyst nematode, Patent application No. PCT/NL92/00126

Ochola J, Cortada L, Ng'ang'a M et al (2020) mediation of potatopotato cyst nematode, $G$. rostochiensis interaction by specific root exudate compounds. Front Plant Sci 11:649. https://doi.org/10. 3389/fpls.2020.00649

Perry RN, Moens M, Jones JT (2018) Cyst nematodes. CABI, Oxfordshire

Sakata I, Kushida A, Tanino K (2020) The hatching-stimulation activity of solanoeclepin A toward the eggs of Globodera (Tylenchida: Heteroderidae) species. Appl Entomol Zool. https://doi.org/10. 1007/s13355-020-00707-5

Sasaky-Crawley A, Curtis R, Birkett M, et al (2010) Signalling and behaviour of Globodera pallida in the rhizosphere of the trap crop Solanum sisymbriifolium. Aspects of Applied Biology, 3rd Symposium on Potato Cyst Nematodes vol 103

Schenk H, Driessen RAJ, Gelder R (1999) Elucidation of the structure of solanoeclepin $\mathrm{A}$, a natural hatching factor of potato and tomato cyst nematodes, by single-crystal X-ray diffraction. Croat Chem Acta 72:593-606

Scholte K (2000a) Effect of potato used as a trap crop on potato cyst nematodes and other soil pathogens and on the growth of a subsequent main potato crop. Ann Appl Biol 136:229-238. https:// doi.org/10.1111/j.1744-7348.2000.tb00029.x

Scholte K (2000b) Screening of non-tuber bearing solanaceae for resistance to and induction of juvenile hatch of potato cyst nematodes and their potential for trap cropping. Ann Appl Biol 136:239-246. https://doi.org/10.1111/j.1744-7348.2000.tb00030.x

Shakya R, Navarre DA (2008) LC-MS analysis of solanidane glycoalkaloid diversity among tubers of four wild potato species and three cultivars (Solanum tuberosum). J Agric Food Chem 56:6949-6958. https://doi.org/10.1021/jf8006618

Shimizu K, Kushida A, Akiyama R et al (2020) Hatching stimulation activity of steroidal glycoalkaloids toward the potato cyst nematode, Globodera rostochiensis. Plant Biotechnol 37:319-325. https://doi.org/10.5511/plantbiotechnology.20.0516a

Stobiecki M, Matysiak-Kata I, Frański R et al (2003) Monitoring changes in anthocyanin and steroid alkaloid glycoside content in lines of transgenic potato plants using liquid chromatography/ mass spectrometry. Phytochemistry 62:959-969. https://doi.org/ 10.1016/S0031-9422(02)00720-3

Tanino K, Takahashi M, Tomata Y et al (2011) Total synthesis of solanoeclepin A. Nat Chem 3:484-488. https://doi.org/10.1038/ nchem. 1044

Urbanová T, Tarkowská D, Novák O et al (2013) Analysis of gibberellins as free acids by ultra performance liquid 
chromatography-tandem mass spectrometry. Talanta 112:85-94. Publisher's Note Springer Nature remains neutral with regard to https://doi.org/10.1016/j.talanta.2013.03.068 jurisdictional claims in published maps and institutional affiliations. 